\title{
Desigualdades sociais em indicadores de envelhecimento ativo: estudo de base populacional
}

\author{
Social inequalities in indicators of active aging: \\ a population-based study
}

Neuciani Ferreira da Silva Sousa (https://orcid.org/0000-0002-7694-0811) ${ }^{1}$

Margareth Guimarães Lima (https://orcid.org/0000-0001-6996-0745) ${ }^{2}$

Marilisa Berti de Azevedo Barros (https://orcid.org/0000-0003-3974-195X) ${ }^{2}$
${ }^{1}$ Departamento de Saúde Coletiva, Instituto de Saúde Coletiva, Universidade Federal de Mato Grosso. R. Quarenta e Nove 2367, Boa Esperança. 78060900 Cuiabá MT Brasil. neuciani@yahoo.com.br ${ }^{2}$ Departamento de Saúde Coletiva, Faculdade de Ciências Médicas, Universidade Estadual de Campinas. Campinas SP Brasil.

\begin{abstract}
The objective of this study was to analy$z e$ inequalities in active aging indicators according to race/skin color, level of education, income, and possession of health insurance among 986 older people who participated in the 2014/15 Campinas Health Survey. We estimated the prevalence of participation in 11 activity domains using Poisson regression. The findings reveal similar levels of participation among white and black people in all the domains of the social dimension. The prevalence of work-related physical activity was higher among black people (14.1\% compared to $8.2 \%$ in white people) and the prevalence of internet use was higher among white people $(P R=2.11)$. The prevalence of participation in leisure time physical activity, internet use, courses, and in all domains of the social dimension except attendance at religious services was higher among respondents in the highest educational and income groups and among those with health insurance. The findings reveal that older people with a higher income and higher level of education are more likely to participate in activities associated with better health and well-being. The study also shows that older people place a significant demand on Brazil's public health system since individuals who depend exclusively on public health services tend to participate less in activities that are shown to promote health benefits.
\end{abstract}

Key words Social inequality, Disparities in health status, Aging, Older people
Resumo Oobjetivo deste estudo foi analisar desigualdades em indicadores de envelhecimento ativo, segundo raça/cor, escolaridade, renda e posse de plano de saúde entre 986 idosos participantes do Inquérito de Saúde de Campinas, São Paulo 2014/2015. Estimaram-se as prevalências de participação dos idosos em 11 atividades e as razões de prevalência foram calculadas pela regressão de Poisson. Os resultados revelaram que brancos $e$ negros participavam de forma semelhante de todas as atividades da dimensão social, porém na atividade fisica realizada no trabalho se observou predominio de negros (14,1\% versus $8,2 \%$ ), e no uso da Internet se constatou maior prevalência de brancos $(R P=2,11)$. Entre os idosos com maior escolaridade, renda mais elevada e posse de plano de saúde foram observadas maiores prevalências de participação em atividades físicas de lazer, uso da Internet, realização de cursos e atividades sociais, exceto cultos religiosos. Os resultados revelam que os idosos com maior acúmulo de recursos educacionais e financeiros têm mais acesso às atividades que são reconhecidamente associadas à saúde e ao bem-estar. O estudo também identificou importantes demandas para o SUS, pois a população que depende exclusivamente desse sistema apresentou menor participação em atividades benéficas à saúde.

Palavras-chave Desigualdade social, Disparidades nos niveis de saúde, Envelhecimento, Idoso 


\section{Introdução}

A relação entre envelhecimento e vida ativa remonta às décadas de 1950 e 1960 nos Estados Unidos, em função da teoria da atividade ${ }^{1}$. Entretanto, foi a Organização Mundial da Saúde (OMS) que transformou a ideia de "envelhecimento ativo" em um conceito de política global ${ }^{2}$, status este sancionado em 2002 no Plano de Ação Internacional de Madrid sobre o envelhecimento $^{3,4}$. Como era de se esperar, o conceito de envelhecimento ativo promovido pela OMS enfatiza a relação entre atividade e saúde ${ }^{2,3}$. E por ter sido desenvolvido em contexto europeu, enfatizou a participação e inclusão dos idosos como cidadãos plenos e favoreceu uma abordagem com foco em uma gama mais ampla de atividades do que aquelas normalmente associadas ao envelhecimento ativo no cenário estadunidense, tais como as atividades produtivas ${ }^{5}$.

Na abordagem da OMS, o termo "ativo" se refere à participação contínua nas questões sociais, econômicas, culturais, físicas, políticas e civis ${ }^{2}$, isto é, considera tanto a participação econômica dos indivíduos quanto outras formas não remuneradas e não produtivas, abarcando atividades formais ou informais, ou que exigem esforço físico ou mental ${ }^{2,3}$. A OMS adotou a perspectiva de que envelhecer ativamente é um direito atrelado às oportunidades ofertadas de saúde, participação, segurança e aprendizagem ao longa da vida, e não simplesmente uma escolha propriamente dita $^{6}$. Isso porque as possibilidades de escolha autônoma são afetadas pelas desigualdades desenfreadas, diferentes experiências de vida e situações sociais e econômicas opressoras ${ }^{7}$. Sendo assim, o risco de se tornar coercitivo pode ser evitado se a política assumir um papel facilitador, com respostas para as diferenças de gênero, raça, classe social e cultura, entre outras, acumuladas ao longo do ciclo de vida.

Diante disso, os estudos sobre envelhecimento ativo deveriam dedicar especial atenção em entender como as diferenças sociais podem dificultar ou diferenciar a vida ativa ${ }^{8,9}$. Porém, os estudos sobre essa temática têm dedicado maior esforço a analisar a associação entre a participação em atividades e desfechos relacionados à saúde, tais como bem-estar subjetivo, saúde física e emocional e sobrevida ${ }^{10}$. Apesar da importância desses estudos com enfoque na saúde, desconsiderar as diferenças sociais na abordagem do envelhecimento ativo é correr o risco de torná-lo um operador de exclusão social e de desigualdades em saúde, posto que nem todos terão condições de aderir à sua proposta de igual maneira ${ }^{8}$. Os estudos que existem nessa perspectiva apontam para uma relação positiva entre nível socioeconômico e participação em atividades socioculturais ${ }^{11-13} \mathrm{e}$ trabalho remunerado ${ }^{14,15}$, porém não há clareza sobre sua relação com a atividade física, por exemplo, que pode variar em função do domínio considerado ${ }^{16,17}$. Além disso, tem sido pouco explorada na literatura a participação em atividades de aprendizagem ou intelectuais segundo estratos sociais ${ }^{18,19}$, bem como os reais efeitos da raça/cor na participação ativa na sociedade, que podem se relacionar tanto ao efeito direto da discriminação racial como ao efeito indireto das diferenças socioeconômicas que permeiam esses estratos. E há uma carência de estudos, nacionais e internacionais, a respeito da contribuição dos serviços de saúde na promoção da vida ativa em múltiplos domínios.

Considerando que as pesquisas sobre envelhecimento ativo deram pouca atenção ao enfoque das desigualdades sociais ${ }^{8,9}$ e que no Brasil a investigação das desigualdades em múltiplas dimensões do envelhecimento ativo em um mesmo estudo é limitada à análise das diferenças de gênero e idade ${ }^{20,21}$, o presente estudo teve por objetivo analisar desigualdades em indicadores de envelhecimento ativo pertencentes às dimensões social, física, intelectual e do trabalho, segundo raça/cor, escolaridade, renda e filiação a plano privado de saúde na população idosa de um município do Sudeste brasileiro.

\section{Métodos}

Trata-se de um estudo transversal, de base populacional, que utilizou dados do Inquérito de Saúde do Município de Campinas (ISACAMP), realizado em 2014 e 2015. Esse inquérito teve por objetivo analisar padrões, tendências e disparidades sociais em múltiplas dimensões da saúde de pessoas com dez anos ou mais residentes em domicílios particulares permanentes da área urbana do município de Campinas-SP.

A seleção da amostra do ISACAMP considerou um desenho complexo de amostragem, em que, primeiramente, foi estabelecida a divisão da população em cinco estratos referentes aos distritos de saúde de Campinas: Leste, Noroeste, Norte, Sudoeste e Sul. Posteriormente, foi realizado o sorteio de conglomerados em dois estágios: setores censitários e domicílios. Em cada estrato foram sorteados 14 setores censitários, totalizando 70 unidades. As populações dos gru- 
pos etários de 10 a 19 anos, 20 a 59 anos e de 60 anos ou mais constituíram domínios de estudo. O cálculo do tamanho da amostra resultou em 3.400 indivíduos, subdivididos em 1.000 adolescentes, 1.400 adultos e 1.000 idosos. Esse número de entrevistas foi previsto de modo a garantir estimativas de proporção de 0,50 , com erro de até cinco pontos percentuais, com intervalos de $95 \%$ de confiança (IC 95\%), considerando um efeito de delineamento de dois. Para obter esse tamanho de amostra, e considerando uma taxa de não resposta esperada de $27 \%, 22 \%$ e $20 \%$ para cada grupo de idade, respectivamente, definidas com base em inquérito anterior, a amostra de domicílios sorteada foi de 3.119 para os adolescentes, 1.029 para os adultos e 3.157 para os idosos.

Em cada domicílio foram entrevistados todos os moradores do grupo de idade para o qual o domicílio havia sido sorteado. A opção pelo não sorteio de indivíduos em cada domicílio se deve ao fato de que este tipo de delineamento é similar em termos de acurácia e tem menor custo em relação ao que prevê o sorteio de uma pessoa por domicílio $^{22}$. Este estudo analisou apenas as pessoas com 60 anos ou mais de idade.

Para cada indivíduo da amostra, foi calculado um peso final, resultado da multiplicação do peso do delineamento pelo peso da taxa de não resposta e o peso do ajuste de pós-estratificação, considerando a distribuição por sexo e idade da projeção populacional elaborada pelo Sistema Estadual de Análise de Dados do Estado de São Paulo - SEADE.

Os dados do ISACAMP 2014/2015 foram coletados por meio de questionário pré-codificado, que continha questões predominantemente fechadas e organizadas em 13 blocos temáticos. A coleta de dados foi feita por entrevistadores treinados, por meio de entrevista direta aos indivíduos, otimizada pela utilização de tablet. Para tanto, os entrevistadores participaram de um programa de treinamento teórico e prático com a finalidade de discutir a conduta esperada durante as entrevistas e os detalhes de cada questão do instrumento, bem como de possibilitar o correto manuseio do tablet e a apropriação e domínio dos conteúdos abordados. Todas as instruções também foram disponibilizadas em um manual.

Em relação às variáveis do estudo, o envelhecimento ativo foi estruturado em consonância com a definição estabelecida pela Organização Mundial da Saúde 2 para o termo "ativo", que diz respeito à participação na sociedade em atividades de múltiplas dimensões, tais como as sociais, físicas, culturais, intelectuais, econômicas, cí- vicas e políticas. Dessa forma, entre as questões disponíveis no questionário, foram selecionadas aquelas pertencentes a quatro dessas dimensões, conforme descrição a seguir:

Dimensão social - os idosos foram indagados sobre a participação atual em quatro domínios: a) visitas ao círculo familiar, por meio da questão " $\mathrm{O}(\mathrm{a})$ sr.(a) recebe ou faz visita a seus amigos e familiares?"; b) atividades socioculturais, a partir da questão "O(a) sr.(a) participa de atividades culturais ou sociais (exemplo: cinema teatro, centros de convivência de idosos, bingos, clubes, baile da terceira idade, festas, entre outros)?”; c) grupos ou associações, considerando a pergunta “ $\mathrm{O}(\mathrm{a})$ sr.(a) faz trabalho voluntário ou faz parte de alguma associação ou grupo esportivo, cultural, filantrópico, político ou religioso?"; d) religião - se frequentava local de culto uma vez por semana ou mais.

Dimensão da atividade física - estimada a partir de questões do Questionário Internacional de Atividades Físicas (IPAQ), versão longa ${ }^{23}$. Esse instrumento estima o tempo semanal gasto na realização de atividades físicas moderadas e/ou vigorosas nos domínios do trabalho, transporte, atividades domésticas e de lazer. Para classificar o nível de atividade física em cada um desses domínios, foi construído um escore de atividade física em minutos por semana a partir da soma dos minutos despendidos em atividades moderadas mais os despendidos em atividades de intensidade vigorosa, sendo nesse último caso multiplicados por dois. Essa estratégia visou considerar as diferentes intensidades de cada atividade, conforme recomenda a $\mathrm{OMS}^{24}$. Um escore acima de 150 minutos por semana foi definido como ponto de corte para classificar os indivíduos como ativos no nível global de atividade física (independente do domínio) e em cada domínio específico (trabalho, deslocamento, domicílio e lazer). Essa diferenciação da prática de atividade física por domínios é importante para identificar e esclarecer características individuais que podem se associar de modo diferencial aos níveis de atividade física em distintos domínios ${ }^{16}$.

Dimensão intelectual - foi estimada a partir de duas variáveis: a) se usavam a Internet; b) realização de cursos, obtida por meio de duas questões: “O(a) sr.(a) frequenta atualmente algum curso regular em escola ou universidade/ faculdade?" e "Atualmente, o(a) sr.(a) frequenta algum outro tipo de curso, como informática, idiomas, dança, artes etc.?”. A variável, de natureza dicotômica, considerou a resposta afirmativa a qualquer uma dessas questões. 
Dimensão do trabalho - verificou-se a participação dos idosos nos seguintes domínios: a) trabalho remunerado: estimada a partir da questão: "Atualmente, o(a) sr.(a) exerce alguma atividade de trabalho remunerado ou ajuda algum familiar na atividade dele?"; b) aposentado que trabalha: idosos em trabalho remunerado, mas que já eram aposentados.

A fim de caracterizar a população de estudo, foram consideradas as seguintes variáveis demográficas e socioeconômicas: sexo (feminino e masculino); idade em faixas etárias (60-69, 70-79, 80 anos ou mais); raça/cor autorreferidas (branca, preta/parda, outras); escolaridade em anos de estudo ( 0 a 3, 4 a 7, 8 ou mais), renda familiar mensal em salários mínimos per capita $(<$ 1,1 a $3,>3$ ) e posse de plano privado de saúde.

Foram estimadas as prevalências de cada domínio do envelhecimento ativo segundo raça/ cor autorreferidas, escolaridade, renda e posse de plano de saúde. Por causa da heterogeneidade e pequeno tamanho da amostra do grupo "outras" (indígena e amarelos) para a variável raça/ cor, foram considerados apenas brancos, pretos e pardos nessa análise. Para a comparação entre as proporções, foi realizado o teste do quiquadrado de Pearson com ajuste de Rao-Scott, considerando-se estatisticamente significativos aqueles com $p<0,05$. As razões de prevalências de cada indicador do envelhecimento ativo, bem como seus respectivos intervalos de confiança de $95 \%$, foram calculados com o uso da regressão de Poisson e ajustados por sexo e idade para eliminar possíveis confundimentos presentes nas associações. As análises segundo raça/cor também foram ajustadas pela escolaridade, a fim de verificar se associações com os indicadores de envelhecimento ativo, caso presentes, poderiam ser explicadas por fatores socioeconômicos ou atribuídas ao efeito direto da discriminação racial no acesso desigual às atividades em questão.

O efeito do desenho amostral complexo foi considerado em todas as análises realizadas, utilizando-se o módulo survey do software Stata 14.0 (Stata Corp., College Station, Estados Unidos).

O ISACAMP foi aprovado pelo Comitê de Ética da Faculdade de Ciências Médicas da Universidade Estadual de Campinas.

\section{Resultados}

Nos domicílios sorteados, foram identificados 1.168 indivíduos com 60 anos ou mais de idade, porém houve $14,0 \%$ de recusa e $1,5 \%$ de perdas por outros motivos, resultando em uma amostra final constituída por 986 idosos. As taxas de não resposta diferiram entre os estratos, tendendo a ser mais elevadas naqueles de melhor nível socioeconômico. Por exemplo, as taxas de não resposta para as entrevistas no domínio dos idosos variaram de 6,3 a $22,6 \%$ entre os distritos de saúde, sendo de $22,6 \%$ no Leste e $18,6 \%$ no Norte, que apresentam melhor nível socioeconômico; e $13,7 \%$ no Sul, $13,1 \%$ no Sudoeste e $6,3 \%$ no Noroeste, em que os residentes têm pior nível socioeconômico. Para reduzir o efeito dessas diferenças, foram utilizados pesos pós-estratificação.

De acordo com a Tabela 1, os idosos eram, em sua maioria, do sexo feminino $(57,6 \%)$, da faixa etária de 60 a 69 anos (56,7\%) e brancos (71,2\%). Além disso, houve predomínio de idosos com menos de oito anos de estudo (65,3\%), com renda familiar per capita de um a três salários mínimos $(55,3 \%)$ e sem posse de plano privado de saúde $(52,9 \%)$.

A análise do perfil de envelhecimento ativo segundo raça/cor da pele (Tabela 2) revelou semelhança na participação de brancos e negros em todas as atividades da dimensão social. Quanto à dimensão da atividade física, considerando a análise ajustada por sexo e idade, apenas no domínio do trabalho verificou-se predomínio dos negros ( $14,1 \%$ versus $8,2 \%)$, e na dimensão intelectual foi observada maior prevalência de uso da Internet entre os brancos ( $\mathrm{RP}=2,11)$. Entretanto, tais diferenças não se mantiveram após o ajuste pela escolaridade. Por outro lado, esse mesmo ajuste revelou menor prevalência de brancos que faziam algum curso ou que exerciam trabalho remunerado.

A Tabela 3 mostra as prevalências de indicadores de envelhecimento ativo segundo o nível de escolaridade. Quanto à dimensão social, apenas a frequência semanal aos cultos religiosos não apresentou diferença entre os estratos de escolaridade. Por outro lado, comparados aos idosos com menor escolaridade, aqueles com oito anos ou mais de estudo apresentaram maiores prevalências de participação no círculo familiar $(\mathrm{RP}=$ $1,10)$, em atividades socioculturais $(\mathrm{RP}=2,30)$ e em voluntariado/associações $(\mathrm{RP}=1,89)$. $\mathrm{Na}$ dimensão da atividade física não houve diferença entre os idosos que possuíam de zero a três e de quatro a sete anos de estudo, porém quando comparados os estratos extremos de escolaridade, observaram-se diferenças nos domínios do trabalho e do lazer, em que os idosos mais escolarizados eram fisicamente mais ativos no lazer (RP $=1,95)$ e menos ativos no trabalho $(\mathrm{RP}=0,62)$. A 
Tabela 1. Distribuição percentual da população idosa segundo características demográficas e socioeconômicas. Campinas - SP, 2014/2015 ( $\mathrm{n}=$ 986).

\begin{tabular}{|c|c|c|}
\hline Variáveis & $\mathbf{n}^{\star}$ & $\%^{* *}$ \\
\hline \multicolumn{3}{|l|}{ Sexo } \\
\hline Masculino & 387 & $42,4(39,6-45,3)$ \\
\hline Feminino & 599 & $57,6(54,7-60,4)$ \\
\hline \multicolumn{3}{|l|}{ Faixa etária (em anos) } \\
\hline 60 a 69 & 506 & $56,7(52,4-60,9)$ \\
\hline 70 a 79 & 308 & $28,0(25,1-31,0)$ \\
\hline 80 e mais & 172 & $15,3(12,5-18,1)$ \\
\hline \multicolumn{3}{|l|}{ Raça/cor } \\
\hline Brancos & 703 & $71,2(66,0-76,5)$ \\
\hline Negros (pretos e pardos) & 254 & $26,1(20,7-31,5)$ \\
\hline Outros & 29 & $2,6(1,4-3,8)$ \\
\hline \multicolumn{3}{|l|}{$\begin{array}{l}\text { Escolaridade (em anos de } \\
\text { estudo) }\end{array}$} \\
\hline 0 a 3 & 341 & $31,9(27,2-36,7))$ \\
\hline 4 a 7 & 342 & $33,4(29,5-37,5)$ \\
\hline 8 ou mais & 303 & $34,6(29,4-39,8)$ \\
\hline \multicolumn{3}{|l|}{$\begin{array}{l}\text { Renda familiar mensal } \\
\left.(\mathrm{em} \mathrm{smpc})^{\mathrm{a}}\right)\end{array}$} \\
\hline$<1$ & 284 & $27,4(22,3-32,6)$ \\
\hline 1 a 3 & 552 & $55,3(50,2-60,5)$ \\
\hline$>3$ & 149 & $17,2(12,2-22,3)$ \\
\hline \multicolumn{3}{|l|}{ Plano de saúde ${ }^{a}$} \\
\hline Sim & 449 & $47,1(40,5-53,7)$ \\
\hline Não & 536 & $52,9(46,3-59,5)$ \\
\hline \multicolumn{3}{|c|}{$\begin{array}{l}\text { * Número de indivíduos na amostra não ponderada; }{ }^{* *} \\
\text { prevalências e intervalos de } 95 \% \text { de confiança (IC } 95 \% \text { ) } \\
\text { calculados sob ponderações devidas ao desenho amostral; }{ }^{a} \\
\mathrm{n}=985 \text { (um indivíduo sem informação); }{ }^{\mathrm{b}} \mathrm{smpc}=\text { salários } \\
\text { mínimos per capita. }\end{array}$} \\
\hline
\end{tabular}

Fonte: Elaborado pelos autores a partir dos dados do Inquérito de Saúde do Município de Campinas (ISACAMP).

dimensão intelectual foi a que apresentou maior discrepância entre os estratos mais baixo e mais alto de escolaridade, mas com estimativas menos precisas. Na dimensão do trabalho não foram observadas diferenças entre os níveis de escolaridade, exceto quanto à prevalência de idosos aposentados que trabalhavam, que foi maior no grupo com escolaridade intermediária, quando comparado ao de baixa escolaridade $(\mathrm{RP}=2,51)$.

De acordo com a Tabela 4, os idosos que possuíam maior renda eram mais ativos socialmente no círculo familiar $(\mathrm{RP}=1,13)$, em atividades socioculturais $(\mathrm{RP}=2,20)$ e em voluntariado/ associações $(R P=2,14)$, bem como intelectualmente, nos dois domínios investigados. Quanto ao trabalho remunerado, não foram observadas diferenças entre os estratos de renda, porém verificou-se maior prevalência de idosos que trabalhavam mesmo já sendo aposentados entre aqueles com maior renda.

Entre os afiliados a plano privado de saúde, observou-se maior participação na dimensão social, exceto na frequência a cultos religiosos, e na dimensão intelectual, tanto no uso da Internet $(\mathrm{RP}=3,75)$ quanto na realização de cursos $(\mathrm{RP}=$ $4,11)$. Na dimensão da atividade física, observouse semelhança entre os grupos nos domínios do trabalho, do deslocamento e do domicílio, mas no domínio do lazer foi observada maior prevalência de participação entre os beneficiários de planos de saúde do que entre aqueles que dependiam exclusivamente do Sistema Único de Saúde - SUS $(R P=1,89)$. Em relação à dimensão do trabalho, não foram observadas diferenças entre os grupos. Esses dados estão na Tabela 5 .

\section{Discussão}

No presente estudo foram apresentados os resultados do Inquérito de Saúde de Campinas sobre a prevalência de participação dos idosos em 11 atividades referentes às dimensões social, intelectual, da atividade física e do trabalho. A pesquisa apontou que brancos e negros participavam igualmente das atividades analisadas, exceto quanto à atividade física no trabalho, mais prevalente entre os negros, e ao uso da Internet, maior entre os brancos. De forma geral, os idosos com maior escolaridade, renda mais elevada e que possuíam plano privado de saúde apresentaram maiores prevalências de participação nas dimensões social, intelectual e da atividade física, particularmente, nesse último caso, no domínio do lazer. Além disso, observou-se semelhança, em todos os estratos estudados, na prevalência de idosos que frequentavam cultos religiosos semanalmente, que eram fisicamente ativos no deslocamento e no domićlilo e que estavam inseridos no mercado de trabalho.

Os resultados segundo raça/cor são positivos no que diz respeito à dimensão social, pois brancos e negros participavam de forma semelhante das atividades consideradas, apesar do acúmulo histórico de desvantagens da população negra em relação à branca ${ }^{25-27}$, claramente associado à menor participação em diferentes dimensões da vida social ${ }^{3}$. Por outro lado, as diferenças observadas nas análises segundo raça/cor também evidenciam que a construção de pontes que aproximem as realidades de brancos e negros no país 
Tabela 2. Prevalência e razão de prevalência de indicadores de envelhecimento ativo segundo raça/cor. Campinas - SP, 2014/2015.

\begin{tabular}{|c|c|c|c|c|c|c|c|c|}
\hline \multirow{3}{*}{ Dimensões } & \multicolumn{3}{|c|}{ Prevalência } & \multirow{3}{*}{$\begin{array}{c}\text { Valor } \\
\text { de } \\
p^{\star *}\end{array}$} & \multicolumn{4}{|c|}{ Razão de prevalência ajustada ${ }^{a}$} \\
\hline & \multirow[b]{2}{*}{ Total } & \multicolumn{2}{|c|}{ Raça/cor ${ }^{\star}$} & & \multirow[b]{2}{*}{$\mathbf{R P}^{\mathbf{b}}$} & \multirow[b]{2}{*}{ IC $95 \%$} & \multirow[b]{2}{*}{$\mathbf{R P}^{\mathbf{c}}$} & \multirow[b]{2}{*}{ IC $95 \%$} \\
\hline & & $\begin{array}{c}\text { Negros } \\
(\mathrm{n}=254)\end{array}$ & $\begin{array}{c}\text { Brancos } \\
(n=703)\end{array}$ & & & & & \\
\hline \multicolumn{9}{|l|}{ Social } \\
\hline Círculo familiar & 89,0 & 86,7 & 89,9 & 0,212 & 1,04 & $0,98-1,11$ & 1,02 & $0,96-1,09$ \\
\hline Atividades socioculturais & 40,2 & 35,7 & 41,6 & 0,199 & 1,20 & $0,93-1,53$ & 1,00 & $0,78-1,29$ \\
\hline Voluntariado ou associações & 23,3 & 22,6 & 23,6 & 0,793 & 1,06 & $0,75-1,50$ & 0,92 & $0,65-1,30$ \\
\hline Cultos religiosos & 47,1 & 50,6 & 45,9 & 0,230 & 0,91 & $0,77-1,06$ & 0,93 & $0,79-1,08$ \\
\hline \multicolumn{9}{|l|}{ Atividade física } \\
\hline Trabalho & 9,9 & 14,1 & 8,2 & 0,005 & 0,65 & $0,44-0,96$ & 0,71 & $0,49-1,05$ \\
\hline Transporte & 10,9 & 9,8 & 11,0 & 0,613 & 1,14 & $0,74-1,76$ & 1,25 & $0,80-1,93$ \\
\hline ++Domicílio & 11,4 & 12,3 & 11,0 & 0,667 & 0,92 & $0,56-1,52$ & 0,88 & $0,54-1,43$ \\
\hline Lazer & 25,3 & 22,5 & 25,7 & 0,296 & 1,20 & $0,94-1,54$ & 1,02 & $0,81-1,30$ \\
\hline Global & 48,6 & 52,8 & 47,1 & 0,086 & 1,07 & $0,95-1,20$ & 1,10 & $0,97-1,26$ \\
\hline \multicolumn{9}{|l|}{ Intelectual } \\
\hline Uso da internet & 21,7 & 13,0 & 25,1 & 0,000 & 2,11 & $1,51-2,94$ & 1,18 & $0,83-1,67$ \\
\hline Cursos & 2,6 & 3,4 & 2,4 & 0,482 & 0,71 & $0,27-1,88$ & 0,41 & $0,18-0,98$ \\
\hline \multicolumn{9}{|l|}{ Trabalho } \\
\hline Trabalho remunerado & 22,1 & 27,7 & 19,7 & 0,015 & 0,79 & $0,60-1,04$ & 0,76 & $0,57-0,99$ \\
\hline Aposentado que trabalha & 5,2 & 4,8 & 5,3 & 0,766 & 1,17 & $0,61-2,25$ & 1,04 & $0,53-2,02$ \\
\hline
\end{tabular}

* Excluídos os amarelos e indígenas; ${ }^{* *}$ teste do qui-quadrado de Pearson com ajuste de Rao-Scott; ${ }^{\text {a }}$ categoria de referência: população negra; ${ }^{b}$ razão de prevalência ajustada por sexo e idade por meio da regressão de Poisson; ${ }^{c}$ razão de prevalência ajustada por sexo, idade e anos de estudo por meio da regressão de Poisson.

Fonte: Elaborado pelos autores a partir dos dados do Inquérito de Saúde do Município de Campinas (ISACAMP).

em outras dimensões da vida ainda é um grande desafio ${ }^{28}$. A primeira diferença diz respeito à prática de atividade física no trabalho. Esse indicador tem sido considerado um marcador de desigualdades sociais ${ }^{29}$ e nem sempre está associado a efeitos benéficos sobre a saúde ${ }^{30}$. Uma revisão sistemática revelou, entre outros aspectos, que os indivíduos de menor nível socioeconômico e que se enquadravam em uma ocupação menos valorizada socialmente possuíam níveis mais elevados de atividade física no trabalho ${ }^{29}$. Assim, o maior nível de atividade física no trabalho entre os negros sinaliza a ocupação de postos que exigem menor qualificação profissional e mais força física. Essa é, precisamente, a realidade brasileira do mercado de trabalho, pois os negros são maioria nos setores com as piores condições laborais - agricultura, construção civil e trabalhos domésticos - e também nas posições mais precárias, isto é, sem a garantia de direitos, tais como o registro trabalhista ${ }^{25,28}$. Quanto ao uso da Internet, a diferença racial observada é inquietante, pois além de os idosos serem a população que menos acessa esse serviço no Brasil ${ }^{18}$, o fato de ser negro diminui ainda mais a probabilidade de usá-la. Tal realidade é distinta da observada nos Estados Unidos, por exemplo, em que a desigualdade digital diminuiu consideravelmente nos últimos 18 anos na população adulta. Em 2000, 53\% dos brancos usavam a Internet, contra $38 \%$ dos negros, passando para $89 \%$ e $87 \%$, respectivamente, em $2018^{31}$.

Parte importante das diferenças raciais identificadas no presente estudo pode ser atribuída à desigualdade educacional entre negros e bran$\cos ^{26,32}$, uma vez que as distinções na prática de atividade física do trabalho e no uso da Internet desapareceram após o ajuste pela escolaridade, enquanto outras diferenças (realização de curso e trabalho remunerado) foram evidenciadas, mas desta vez a favor dos negros. Nesse caso, isso não significa, necessariamente, melhor condição da população negra, pois faz-se necessário estudo mais aprofundado sobre possíveis diferenças intrínsecas às atividades em questão.

Em relação às análises segundo o nível socioeconômico, medido tanto pela escolaridade quanto pela renda, verificou-se maior prevalên- 
Tabela 3. Prevalência e razão de prevalência de indicadores de envelhecimento ativo segundo anos de estudo. Campinas - SP, 2014/2015.

\begin{tabular}{|c|c|c|c|c|c|c|c|c|}
\hline \multirow{3}{*}{ Dimensões } & \multirow{2}{*}{\multicolumn{3}{|c|}{$\begin{array}{c}\text { Prevalência } \\
\text { Anos de estudo }\end{array}$}} & \multirow{3}{*}{$\begin{array}{l}\text { Valor } \\
\text { de } p^{\star \star}\end{array}$} & \multicolumn{4}{|c|}{ Razão de prevalência ajustada ${ }^{a}$} \\
\hline & & & & & \multirow{2}{*}{$\begin{array}{l}\mathbf{R P}^{\mathbf{b}} \\
(2 / 1)\end{array}$} & \multirow[b]{2}{*}{ IC $95 \%$} & \multirow{2}{*}{$\begin{array}{l}R^{b} \\
(3 / 1)\end{array}$} & \multirow[b]{2}{*}{ IC 95\% } \\
\hline & $\begin{array}{c}0 \text { a } 3 \\
(n=341)(1)\end{array}$ & $\begin{array}{c}4 \text { a } 7 \\
(n=342)(2)\end{array}$ & $\begin{array}{c}\geq 8 \\
(n=303)(3)\end{array}$ & & & & & \\
\hline \multicolumn{9}{|l|}{ Social } \\
\hline Círculo familiar & 83,6 & 90,6 & 92,5 & 0,005 & 1,08 & $1,02-1,14$ & 1,10 & $1,03-1,17$ \\
\hline $\begin{array}{l}\text { Atividades } \\
\text { socioculturais }\end{array}$ & 24,2 & 36,8 & 58,2 & $<0,001$ & 1,49 & $1,13-1,98$ & 2,30 & $1,81-2,91$ \\
\hline $\begin{array}{l}\text { Voluntariado ou } \\
\text { associações }\end{array}$ & 15,4 & 24,3 & 29,7 & 0,001 & 1,57 & $1,11-2,21$ & 1,89 & $1,28-2,78$ \\
\hline Cultos religiosos & 49,9 & 46,7 & 44,9 & 0,473 & 0,94 & $0,80-1,10$ & 0,89 & $0,75-1,04$ \\
\hline \multicolumn{9}{|l|}{ Atividade física } \\
\hline Trabalho & 9,6 & 10,5 & 9,6 & 0,910 & 0,88 & $0,55-1,40$ & 0,62 & $0,40-0,96$ \\
\hline Deslocamento & 9,6 & 13,8 & 9,1 & 0,156 & 1,33 & $0,81-2,21$ & 0,82 & $0,46-1,47$ \\
\hline Domicílio & 9,5 & 11,6 & 12,5 & 0,656 & 1,12 & $0,67-1,85$ & 1,14 & $0,63-2,08$ \\
\hline Lazer & 15,6 & 23,5 & 36,0 & $<0,001$ & 1,38 & $0,94-2,05$ & 1,95 & $1,32-2,87$ \\
\hline Global & 39,6 & 51,9 & 54,7 & 0,001 & 1,19 & $0,99-1,42$ & 1,13 & $0,93-1,37$ \\
\hline \multicolumn{9}{|l|}{ Intelectual } \\
\hline Uso da internet & 2,2 & 11,0 & 50,1 & $<0,001$ & 4,53 & $2,33-8,80$ & 18,90 & $9,81-36,32$ \\
\hline Cursos & 0,5 & 1,3 & 5,8 & $<0,001$ & 2,71 & $0,51-14,49$ & 13,16 & $2,80-61,96$ \\
\hline \multicolumn{9}{|l|}{ Trabalho } \\
\hline $\begin{array}{l}\text { Trabalho } \\
\text { remunerado }\end{array}$ & 16,8 & 21,0 & 28,0 & 0,008 & 1,04 & $0,74-1,45$ & 1,11 & $0,81-1,52$ \\
\hline $\begin{array}{l}\text { Aposentado que } \\
\text { trabalha }\end{array}$ & 2,4 & 6,7 & 6,5 & 0,025 & 2,51 & $1,22-5,17$ & 2,17 & $0,91-5,15$ \\
\hline
\end{tabular}

Fonte: Elaborado pelos autores a partir dos dados do Inquérito de Saúde do Município de Campinas (ISACAMP).

cia de participação na dimensão social (visitas ao círculo familiar, atividades socioculturais e voluntariado/associações) nos estratos de maior nível. Em outros estudos, o melhor patamar socioeconômico também esteve associado à maior participação social ${ }^{11-14}$. Uma explicação para tais associações é que o maior nível socioeconômico incorpora uma variedade de recursos, como dinheiro, conhecimento, prestígio e poder, que favorecem a produção da saúde, bem como a vida ativa $^{33}$. Por outro lado, a participação social não depende apenas de recursos individuais, mas sobretudo do contexto de desigualdade social em que se está inserido. Nos países onde a desigualdade de renda é menor, por exemplo, o sistema de prestação de serviços à sociedade é mais equânime, facilitando a participação dos menos favorecidos $^{34}$. Como no Brasil a desigualdade social persiste com ampla magnitude ${ }^{27}$, é de se esperar que os segmentos sociais mais vulneráveis en- contrem maior dificuldade de participar de atividades sociais.

Em relação à participação em atividades físicas, verificou-se neste estudo um efeito bidirecional do nível de escolaridade, em que o estrato mais elevado se relacionou de forma direta com a atividade física de lazer e de forma inversa com a prática de atividade física no trabalho. E quanto à renda, verificou-se associação direta com a atividade física global, em especial pela diferença na atividade física de lazer. Uma revisão sistemática também observou diferenças consideráveis na direção das desigualdades entre os distintos domínios de atividade física, em que o nível socioeconômico mais elevado estava associado à maior atividade física durante o tempo de lazer, enquanto a posição socioeconômica mais baixa se associou à maior atividade física ocupacional ${ }^{17}$. Uma das hipóteses é que indivíduos de nível socioeconômico mais elevado são mais prováveis 
Tabela 4. Prevalência e razão de prevalência de indicadores de envelhecimento ativo segundo renda. Campinas SP, $2014 / 2015$.

\begin{tabular}{|c|c|c|c|c|c|c|c|c|}
\hline \multirow{3}{*}{ Dimensões } & \multirow{2}{*}{\multicolumn{3}{|c|}{$\begin{array}{c}\text { Prevalência } \\
\text { Renda em smpc }\end{array}$}} & \multirow[b]{3}{*}{$\begin{array}{l}\text { Valor } \\
\text { de } \mathbf{p}^{\star *}\end{array}$} & \multicolumn{4}{|c|}{ Razão de prevalência ajustada ${ }^{a}$} \\
\hline & & & & & \multirow[b]{2}{*}{$\begin{array}{l}\mathrm{RP}^{\mathrm{b}} \\
(2 / 1)\end{array}$} & \multirow[b]{2}{*}{ IC $95 \%$} & \multirow[b]{2}{*}{$\begin{array}{c}\mathbf{R P}^{\mathrm{b}} \\
(3 / 1)\end{array}$} & \multirow[b]{2}{*}{ IC $95 \%$} \\
\hline & $\begin{array}{c}<1 \\
(n=284)(1)\end{array}$ & $\begin{array}{c}1 \text { a } 3 \\
(n=552)(2)\end{array}$ & $\begin{array}{c}>3 \\
(n=149)(3)\end{array}$ & & & & & \\
\hline \multicolumn{9}{|l|}{ Social } \\
\hline Círculo familiar & 83,5 & 90,5 & 94,6 & 0,003 & 1,08 & $1,01-1,17$ & 1,13 & $1,05-1,22$ \\
\hline $\begin{array}{l}\text { Atividades } \\
\text { socioculturais }\end{array}$ & 25,6 & 41,5 & 58,2 & $<0,001$ & 1,60 & $1,19-2,17$ & 2,20 & $1,60-3,02$ \\
\hline $\begin{array}{l}\text { Voluntariado ou } \\
\text { associações }\end{array}$ & 16,8 & 22,1 & 36,3 & 0,002 & 1,32 & $0,90-1,94$ & 2,14 & $1,38-3,32$ \\
\hline Cultos religiosos & 44,8 & 48,9 & 43,8 & 0,544 & 1,11 & $0,93-1,33$ & 0,99 & $0,73-1,34$ \\
\hline \multicolumn{9}{|l|}{ Atividade física } \\
\hline Trabalho & 7,2 & 10,5 & 12,7 & 0,170 & 1,36 & $0,83-2,24$ & 1,48 & $0,77-2,85$ \\
\hline Deslocamento & 9,6 & 11,8 & 10,1 & 0,636 & 1,20 & $0,76-1,89$ & 0,99 & $0,51-1,91$ \\
\hline Domicílio & 12,3 & 9,7 & 15,4 & 0,259 & 0,78 & $0,48-1,27$ & 1,21 & $0,68-2,15$ \\
\hline Lazer & 13,4 & 26,1 & 40,2 & $<0,001$ & 1,87 & $1,32-2,65$ & 2,74 & $1,99-3,77$ \\
\hline Global & 38,2 & 50,6 & 59,7 & 0,001 & 1,28 & $1,02-1,60$ & 1,43 & $1,17-1,52$ \\
\hline \multicolumn{9}{|l|}{ Intelectual } \\
\hline Uso da internet & 10,8 & 17,6 & 51,2 & $<0,001$ & 1,57 & $1,03-2,39$ & 4,27 & $2,71-6,74$ \\
\hline Cursos & 0,6 & 1,6 & 9,0 & $<0,001$ & 2,74 & $0,55-13,61$ & 15,36 & $3,39-69,60$ \\
\hline \multicolumn{9}{|l|}{ Trabalho } \\
\hline $\begin{array}{l}\text { Trabalho } \\
\text { remunerado }\end{array}$ & 20,3 & 20,9 & 29,2 & 0,173 & 0,97 & $0,69-1,37$ & 1,23 & $0,84-1,79$ \\
\hline $\begin{array}{l}\text { Aposentado que } \\
\text { trabalha }\end{array}$ & 2,2 & 5,5 & 9,2 & 0,012 & 2,40 & $1,04-5,53$ & 3,76 & $1,42-9,92$ \\
\hline
\end{tabular}

${ }^{*}$ smpc = salários mínimos per capita; ${ }^{* *}$ teste do qui-quadrado de Pearson com ajuste de Rao-Scott; ${ }^{a}$ categoria de referência:

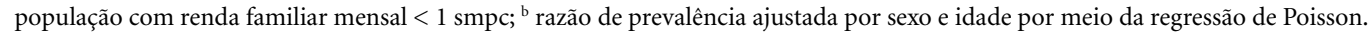

Fonte Elaborado pelos autores a partir dos dados do Inquérito de Saúde do Município de Campinas (ISACAMP).

de aderir aos programas preventivos e comportamentos saudáveis, seja pela maior informação, motivação ou acesso a outros recursos, como clubes esportivos e academias de ginástica ${ }^{17,35}$. Por outro lado, a relação inversa entre atividade física no trabalho e escolaridade possivelmente reflete o acesso a ocupações de menor prestígio social, que implicam níveis mais elevados de gasto energético ${ }^{29}$, a exemplo do que foi discutido anteriormente na análise segundo raça/cor.

Com relação à dimensão intelectual, apesar da menor precisão das estimativas, chama atenção a discrepância observada no uso da Internet entre os estratos de escolaridade e renda, em que os mais desfavorecidos se mostraram significativamente menos propensos a utilizá-la. No Brasil, existe um claro gradiente socioeconômico positivo em relação ao uso da Internet, e tal qual no presente estudo, o gradiente educacional é mais acentuado que o de renda ${ }^{18}$. Esses achados sugerem que um dos determinantes mais fortes do uso da Internet é o nível educacional, pois potencializa a apropriação das tecnologias em rápida transformação. Assim, o uso da Internet pode refletir, cada vez mais, as relações sociais, econômicas e culturais presentes no mundo off-line, incluindo as desigualdades sociais ${ }^{36}$. Portanto, as políticas públicas brasileiras de inclusão digital devem abarcar não apenas os investimentos em compra de equipamentos, a disponibilização do acesso à Internet em locais públicos ou a redução de seu custo em âmbito privado, mas uma contínua melhoria do ensino básico, para que todas as camadas da sociedade tenham condições de explorar, compreender e se apropriar das informações disponibilizadas pela Internet ${ }^{36,37}$.

Ainda em relação à dimensão intelectual, verificou-se baixa prevalência de idosos que frequen- 
Tabela 5. Prevalência e razão de prevalência de indicadores de envelhecimento ativo segundo posse de plano de saúde. Campinas - SP, 2014/2015.

\begin{tabular}{|c|c|c|c|c|c|}
\hline \multirow{3}{*}{ Dimensões } & \multirow{2}{*}{\multicolumn{2}{|c|}{$\begin{array}{c}\text { Prevalência } \\
\text { Plano de saúde }\end{array}$}} & \multirow{3}{*}{$\begin{array}{c}\text { Valor de } \\
\mathrm{p}^{\star}\end{array}$} & \multicolumn{2}{|c|}{ Razão de prevalência ajustada ${ }^{a}$} \\
\hline & & & & \multirow[b]{2}{*}{$\mathbf{R} \mathbf{P}^{\mathbf{b}}$} & \multirow[b]{2}{*}{ IC $95 \%$} \\
\hline & $\begin{array}{c}\text { Não } \\
(n=536)\end{array}$ & $\begin{array}{c}\text { Sim } \\
(\mathrm{n}=449)\end{array}$ & & & \\
\hline \multicolumn{6}{|l|}{ Social } \\
\hline Círculo familiar & 86,4 & 91,9 & 0,014 & 1,07 & $1,02-1,12$ \\
\hline Atividades socioculturais & 32,5 & 48,9 & $<0,001$ & 1,56 & $1,30-1,87$ \\
\hline Voluntariado ou associações & 18,0 & 29,2 & 0,001 & 1,65 & $1,23-2,21$ \\
\hline Cultos religiosos & 45,9 & 48,3 & 0,530 & 1,04 & $0,88-1,23$ \\
\hline \multicolumn{6}{|l|}{ Atividade física } \\
\hline Trabalho & 10,8 & 9,0 & 0,393 & 1,00 & $0,66-1,51$ \\
\hline Deslocamento & 10,2 & 11,5 & 0,564 & 1,19 & $0,77-1,84$ \\
\hline Domicílio & 11,4 & 11,2 & 0,939 & 1,01 & $0,67-1,54$ \\
\hline Lazer & 18,8 & 32,6 & $<0,001$ & 1,89 & $1,57-2,29$ \\
\hline Global & 46,1 & 52,1 & 0,083 & 1,22 & $1,08-1,37$ \\
\hline \multicolumn{6}{|l|}{ Intelectual } \\
\hline Uso da internet & 10,4 & 34,5 & $<0,001$ & 3,75 & $2,65-5,30$ \\
\hline Realização de cursos & 1,1 & 4,4 & 0,004 & 4,11 & $1,41-12,0$ \\
\hline \multicolumn{6}{|l|}{ Trabalho } \\
\hline Trabalho remunerado & 25,0 & 18,9 & 0,047 & 0,88 & $0,67-1,16$ \\
\hline Aposentado que trabalha & 5,2 & 5,2 & 0,979 & 1,11 & $0,65-1,89$ \\
\hline
\end{tabular}

Fonte: Elaborado pelos autores a partir dos dados do Inquérito de Saúde do Município de Campinas (ISACAMP).

tavam algum tipo curso, e apesar das estimativas pouco precisas, os dados evidenciam que os mais favorecidos em termos educacionais e financeiros tinham mais probabilidade de participar de atividades de aprendizagem, coadunando com a realidade nacional ${ }^{32}$. No cenário internacional, uma análise feita a partir dos resultados de 13 estudos longitudinais e de dois transversais de diferentes países revelou um padrão relativamente claro de que os indivíduos em melhor situação socioeconômica tiveram melhores condições para acessar a educação na fase adulta e usufruir de mais benefícios no progresso da carreira. O estudo concluiu que a aprendizagem de adultos tende a reproduzir e reforçar os resultados da educação inicial, ou seja, existe uma espécie de seletividade educacional dos participantes, em que aqueles que possuem maior nível de escolaridade são também mais propensos a participar de outros processos de aprendizagem durante seu curso de vida $^{19}$. Nessa perspectiva, os achados do presente estudo reiteram o argumento de que deve ser dada mais atenção à complexa relação entre desigualdade social e aprendizagem na vida adulta ou velhice $^{19}$, a fim de evitar que a educação se torne, cada vez mais, uma mercadoria, e não um direito.

Quanto à participação no mercado de trabalho, surpreende o fato de que não houve diferença entre os estratos socioeconômicos, uma vez que é mais comum uma relação direta e gradual entre essas variáveis, principalmente quando considerado o nível de escolaridade ${ }^{14,15}$. A relação entre recursos financeiros e trabalho é menos clara, pois aqueles com baixos níveis de renda podem ter que trabalhar para manter um padrão mínimo de vida, enquanto, no extremo oposto, aqueles com altos níveis de renda também têm mais chances de ter melhor educação, e dado que a educação está positivamente relacionada ao emprego, eles são mais propensos a permanecerem empregados ${ }^{15,38}$. Porém, isso não exclui a possibilidade de que aqueles com níveis de renda suficientes para manter uma vida digna e satisfatória optem por deixar o trabalho assim que possível, de modo a aproveitar a velhice participando de outros tipos de atividades. Cabe reforçar, no entanto, que apesar de não terem sido identificadas diferenças entre os estratos socioe- 
conômicos na participação em trabalho remunerado, os resultados do presente estudo devem ser analisados com cautela, pois o indicador utilizado não apreende outros aspectos do mercado de trabalho que podem ser desiguais entre os distintos estratos sociais ${ }^{14,15,38}$.

Em relação à análise segundo filiação a plano privado de saúde, o presente estudo encontrou que a população beneficiária de planos era mais ativa na dimensão social e intelectual e na prática de atividade física de lazer. No Brasil, essa análise é particularmente importante, pois expõe a demanda para aqueles que dependem exclusivamente do SUS. Isso porque há uma expectativa de que as instituições que prestam serviços aos idosos não se dediquem apenas a atender suas necessidades físicas, mas avancem no direcionamento de serviços que conectem os idosos à sua comunidade ${ }^{39}$. A participação social é altamente valorizada pelos idosos e deve ser promovida no âmbito do SUS, tendo em vista seu potencial para promover a saúde física e mental dos idosos, bem como gerar benefícios sociais por meio do aumento das contribuições comunitárias deste grupo ${ }^{12}$. A promoção do voluntariado e a criação de grupos comunitários que desenvolvam atividades de natureza cultural, lúdica e educacional são exemplos de medidas que podem ser fomentadas no âmbito do SUS ${ }^{39}$, conforme previsto na Política Nacional de Saúde da Pessoa Idosa ${ }^{40}$. Essas ações são particularmente importantes para promover a inclusão social e tecnológica e otimizar a conexão social, o desenvolvimento de habilidades adicionais e a preservação das funções cognitivas ${ }^{3}$. Entretanto, independentemente do que o SUS possa proporcionar, faz-se necessário também o enfrentamento de barreiras que limitam a participação social dos idosos, como a falta de tempo livre para o lazer advinda da sobrecarga imposta pelos cuidados familiares, as dificuldades de mobilidade urbana e a falta de orientação para o lazer ${ }^{41}$.

Outro aspecto revelado pelo estudo diz respeito à desigualdade na prática de atividade física global, sobretudo pela diferença em relação à atividade física de lazer entre a população com e sem plano de saúde. Isso reforça a relevância de programas como a Academia da Saúde e da atuação efetiva dos Núcleos de Apoio à Saúde da Família na ampliação do acesso a práticas corporais e atividade física ${ }^{42,43}$. E a fim de favorecer a participação de subgrupos de idosos que se diferenciam em função do gênero, faixa etária, nível de saúde, disponibilidade de tempo e preferências individuais $^{44}$, torna-se necessária a ampliação do escopo e das modalidades oferecidas nesses espaços ${ }^{42}$.
O presente estudo apresentou algumas limitações, como a ausência de informações sobre a frequência de realização das atividades socioculturais e da participação em grupos ou associações e os motivos e a frequência de uso da Internet. Tais detalhes ajudariam a compreender melhor o objeto em questão, pois a realização das atividades pode ter efeitos divergentes na saúde e no bem-estar, a depender do contexto em que são realizadas. O viés de sobrevivência foi considerado outra limitação desta pesquisa, visto que os segmentos de pior nível socioeconômico têm maior risco de mortalidade precoce e, portanto, menor probabilidade de serem incluídos no estudo. Dessa forma, esse viés tende a reduzir a magnitude das associações encontradas entre as variáveis de estudo $^{45}$. As forças do estudo se devem ao uso de uma base populacional, ao tamanho da amostra, que permitiu estimar a maioria dos indicadores de envelhecimento ativo com bom nível de precisão nos estratos considerados, à qualidade dos dados coletados e ao uso de indicadores pouco explorados para a população idosa, partindo-se de uma abordagem multidimensional do envelhecimento ativo.

\section{Considerações finais}

Este estudo revelou a magnitude das desigualdades sociais que permeiam os múltiplos indicadores de envelhecimento ativo, contribuindo para ampliar a visão sobre essa temática, e discutiu os desafios no que concerne à sua promoção. A pesquisa identificou que os idosos com maior acúmulo de recursos educacionais e financeiros continuam tendo mais acesso, na velhice, às atividades que são reconhecidamente associadas a melhor saúde e bem-estar, tais como as atividades da dimensão social, as atividades físicas de lazer e as intelectuais. Entre as desigualdades a serem combatidas, destaca-se a educacional, que conforme discussão realizada no presente estudo, é um importante determinante do envelhecimento ativo, não apenas por potencializar $\mathrm{o}$ acesso às atividades na velhice, mas sobretudo por qualificá-lo.

Especificamente, os achados confirmam a relação positiva entre nível socioeconômico e participação em atividades socioculturais, porém contrapõem sua associação positiva com o trabalho remunerado, instigando futuros estudos sobre a temática. Além disso, o estudo ratifica que a relação entre nível socioeconômico e atividade física varia em função do domínio con- 
siderado, sendo positiva para atividade física no lazer e negativa para atividade física no trabalho. Verificou-se, ainda, que as diferenças observadas segundo raça/cor para a prática de atividade física no trabalho e uso da Internet foram atribuídas ao efeito indireto das diferenças socioeconômicas que permeiam esses estratos, e não ao efeito direto da discriminação racial, que podem ser mais evidentes em outros contextos culturais. Por fim, o estudo também identificou importantes demandas para o SUS, uma vez que a população que depende exclusivamente dele é menos presente em atividades que reconhecidamente promovem benefícios à saúde (atividades inte- lectuais e socioculturais, participação em grupos/ associações e voluntariado e atividades físicas no domínio do lazer). Nessa perspectiva, ressaltase não só a necessidade de promoção de ações específicas de saúde no âmbito do SUS, mas de fomento à participação social como forma de retroalimentar a saúde dos idosos.

Os achados assinalam que a promoção do envelhecimento ativo pressupõe o combate às desigualdades sociais ao longo do curso de vida, com vistas a fornecer soluções mais justas e sensíveis às diferenças existentes entre os segmentos da sociedade, de modo a evitar a permanência e extensão das desigualdades sociais em saúde.

\section{Colaboradores}

NFS Sousa participou da concepção, análise e interpretação dos dados e da redação do texto. MGL colaborou na interpretação dos dados e na revisão crítica do texto. MBA Barros participou da concepção, análise e interpretação dos dados, da revisão crítica do texto e aprovação final da versão a ser publicada.

\section{Agradecimentos}

À Fundação de Amparo à Pesquisa do Estado de São Paulo (FAPESP), pelo financiamento do inquérito ISACAMP. Ao Ministério da Saúde/SVS/ Secretaria Municipal de Saúde de Campinas, por suporte financeiro complementar ao inquérito ISACAMP. À Coordenação de Aperfeiçoamento em Pesquisa (CAPES), pela concessão de bolsa de doutorado a NFS Sousa. Ao Conselho Nacional de Desenvolvimento Científico e Tecnológico $(\mathrm{CNPq})$, pela bolsa de produtividade concedida a MBA Barros.

\section{Referências}

1. Havighrst RJ. Flexibility and the social roles of the retired. Am J Sociol 1954; 59:309-311.

2. World Health Organization (WHO). Active ageing: $a$ policy framework. Geneva: WHO; 2002.

3. Centro Internacional de Longevidade Brasil. Envelhecimento ativo: um marco político em resposta à revolução da longevidade. Rio de Janeiro: Centro Internacional de Longevidade Brasil; 2015.

4. Walker A. A strategy for active ageing. Int Soc Secur Rev 2002; 55(1):121-39.

5. Walker A. Active ageing: realising its potential. Australas J Ageing 2015; 34(1):2-8.

6. United Nations. General Assembly. A/RES/46/91 (Implementation of the International Plan of Action on Ageing and related activities); 1991 Dec 16.

7. Holstein MB, Minkler M. Self, Society, and the "new gerontology". Gerontologist 2003; 43(6):787-796.

8. Timonen V. Beyond successful and active ageing: a theory of model ageing. Bristol: Policy Press; 2016.

9. Barslund $\mathrm{M}$, von Werder $\mathrm{M}$, Zaid $\mathrm{A}$. Inequality in active ageing: evidence from a new individual-level index for European countries. Ageing Soc 2017;39(3):541567.

10. Adams KB, Leibbrandt S, Moon H. A critical review of the literature on social and leisure activity and wellbeing in later life. Ageing Soc 2011; 31(4):683-712.

11. van Groenou MB, Deeg DJH. Formal and informal social participation of the 'young-old' in The Netherlands in 1992 and 2002. Ageing Soc 2010; 30(3):445465.

12. Galenkamp H, Gagliardi C, Principi A, Golinowska S, Moreira A, Schmidt AE, Winkelmann J, Sowa A, van der Pas S, Deeg DJ. Predictors of social leisure activities in older Europeans with and without multimorbidity. Eur J Ageing 2016; 13(2):129-143. 
13. Vézina $\mathrm{M}$, Crompton S. Volunteering in Canada. $\mathrm{Ca}$ nadian Social Trends 2012; 93:37-55.

14. Ilinca S, Rodrigues R, Schmidt A, Zolyomi E. Gender and social class inequalities in active ageing: policy meets theory. Vienna: European Centre for Social Welfare Policy and Research; 2016.

15. Uppal S. Labour market activity among seniors. Perspectives on Labour and Income 2010; 11(7):5-18.

16. Notthoff N, Reisch P, Gerstorf D. Individual characteristics and physical activity in older adults: a systematic review. Gerontology 2017; 63(5): 443-459.

17. Beenackers MA, Kamphuis CB, Giskes K, Brug J, Kunst AE, Burdorf A, van Lenthe FJ . Socioeconomic inequalities in occupational, leisure-time, and transport related physical activity among European adults: a systematic review. Int J Behav Nutr Phys Act 2012; 9:116.

18. Instituto Brasileiro de Geografia e Estatística (IBGE). Pesquisa nacional por amostra de domicílios: acesso à internet e à televisão e posse de telefone móvel celular para uso pessoal 2015. Rio de Janeiro: IBGE; 2016.

19. Kilpi-Jakonen E, Vilhena DV, HP Blossfeld. Adult learning and social inequalities: Processes of equalisation or cumulative disadvantage? Int Rev Educ 2015; 61(4):529-546.

20. Ribeiro PCC, Neri AL, Cupertino APFB, Yassuda MS. Variabilidade no envelhecimento ativo segundo gênero, idade e saúde. Psicol Estud 2009; 14(3):501-509.

21. Sousa NFS, Lima MG, Cesar CLG, Barros MBA. Envelhecimento ativo: prevalência e diferenças de gênero e idade em estudo de base populacional. Cad Saude Publica 2018; 34(11):e00173317.

22. Alves MCGP, Escuder MML, Claro RM, Silva NN. Sorteio intradomiciliar em inquéritos de saúde. Rev Saude Publica 2014; 48(1):86-93.

23. Matsudo S, Araújo T, Matsudo V, Andrade D, Andrade E, Oliveira LC, Braggion G. Questionário internacional de atividade física (IPAQ): estudo de validade e reprodutibilidade no Brasil. Rev Bras Ativ Fís Saúde 2001; 6(2):5-18.

24. World Health Organization (WHO). Global recommendations on physical activity for health. Geneva: WHO; 2010.

25. Instituto de Pesquisa Econômica Aplicada (Ipea), Secretaria Especial de Políticas para as Mulheres, ONU Mulheres. Retrato das desigualdades de gênero e raça. $4^{\mathrm{a}}$ ed. Brasília: Ipea; 2011.

26. Instituto de Pesquisa Econômica Aplicada (Ipea). Retrato das desigualdades de gênero e raça - 1995 a 2015. Brasília: Ipea; 2017.

27. Oxford Committee for Famine Relief Brasil. A distância que nos une: um retrato das desigualdades brasileiras. São Paulo: OXFAM Brasil; 2017.

28. Charão C. O longo combate às desigualdades raciais. Desenvolvimento 2011; 70:22-31.

29. Smith L, McCourt O, Sawyer A, Ucci M, Marmot A, Wardle J, Fischer A. A review of occupational physical activity and sedentary behaviour correlates. Occup Med (Lond) 2016; 66(3):185-192.

30. Abu-Omar K, Rütten A. Relation of leisure time, occupational, domestic, and commuting physical activity to health indicators in Europe. Prev Med 2008; 47(3):319-323.

31. Pew Research Center. Internet use by racelethnicity; 2017. [acesso 2018 Jul 11]. Disponível em: https://www. pewresearch.org/internet/chart/internet-use-by-race/
32. Instituto Brasileiro de Geografia e Estatística (IBGE) Educação 2016. Rio de Janeiro: IBGE; 2017.

33. Phelan JC, Link BG, Tehranifar P. Social conditions as fundamental causes of health inequalities: theory, evidence, and policy implications. J Health Soc Behav 2010; 51(Suppl.):s28-s40.

34. Lancee B, van de Werfhorst HG. Income inequality and participation: a comparison of 24 European countries. Soc Sci Res 2012; 41(5):1166-1178.

35. Del Duca GF, Rombaldi AJ, Knuth AG, Azevedo MR, Nahas MV, Hallal PC. Associação entre nível econômico e inatividade física em diferentes domínios. Rev Bras Ativ Fís Saúde 2009; 14(2):123-131.

36. van Deursen AJAM, van Dijk JAGM. The digital divide shifts to differences in usage. New Media Soc 2014; 16(3):507-526.

37. Mattos FAM, Chagas GJN. Desafios para a inclusão digital no Brasil. Perspect Ciênc Inf 2008; 13(1):67-94.

38. Wajnman S, Oliveira AM, Oliveira EL. Os idosos no mercado de trabalho: tendências e consequências. In: Camarano AA, organizadora. Os novos idosos brasileiros: muito além dos 60? Rio de Janeiro: Ipea; 2004. p. 453-479.

39. Douglas H, Georgiou A, Westbrook J. Social participation as an indicator of successful aging: an overview of concepts and their associations with health. Aust Health Rev 2017; 41(4):455-462

40. Brasil. Portaria $\mathrm{n}^{\circ} 2.528 / \mathrm{MS}$ de 19 de outubro de 2006. Aprova a Política Nacional de Saúde da pessoa idosa. Diário Oficial da União 2206; 20 out.

41. Levasseur M, Généreux M, Bruneau JF, Vanasse A, Chabot E, Beaulac C, Bédard MM. Importance of proximity to resources, social support, transportation and neighborhood security for mobility and social participation in older adults: results from a scoping study. BMC Public Health 2015; 15:503.

42. Carvalho FFB, Nogueira JAD. Práticas corporais e atividades físicas na perspectiva da promoção da saúde na atenção básica. Cien Saude Colet 2016; 21(6):18291838.

43. Fernandes AP, Andrade ACZ, Costa DAS, Dias MAS, Malta DC, Caiaffa WT. Programa Academias da Saúde e a promoção da atividade física na cidade: a experiência de Belo Horizonte, MG, Brasil. Cien Saude Colet 2017; 22(12):3903-3914.

44. Costa TB, Ribeiro LHM, Neri AL. Prevalence of and factors associated with leisure-time physical activity in older adults from seven Brazilian cities: data from the FIBRA study. Rev Bras Ativ Fís Saúde 2015; 20(2):174-83.

45. Lima-Costa MF, Barreto SM. Tipos de estudos epidemiológicos: conceitos básicos e aplicações na área do envelhecimento. Epidemiol Serv Saude 2003; 12(4):189-201.

Artigo apresentado em 18/12/2018

Aprovado em 15/10/2019

Versão final apresentada em 17/10/2019

Editores-chefes: Romeu Gomes, Antônio Augusto Moura da Silva 